\title{
Effects of Hydrogen during Molecular Beam Epitaxy of GaN
}

\author{
Y. Dong ${ }^{1}$ and R. M. Feenstra ${ }^{{ }^{*}}$ \\ ${ }^{1}$ Department of Physics, Carnegie Mellon University, Pittsburgh, Pennsylvania 15213, USA
}

Received $\mathbf{z} \mathbf{z} \mathbf{z}$, revised $\mathbf{z} \mathbf{z}$, accepted $\mathbf{z} \mathbf{z}$

Published online $\mathbf{z} \mathbf{z} \mathbf{z}$

\section{PACS 68.55.Jk, 81.05.Ea, 81.15.Hi}

We study the effect of introducing hydrogen gas through the RF plasma source during plasma-assisted molecular beam epitaxy of $\mathrm{GaN}(0001)$. The well-known smooth-to-rough transition that occurs for this surface as a function of decreasing Ga flux in the absence of $\mathrm{H}$ is found to persist even with $\mathrm{H}$ present. But, the critical Ga flux for this transition is increased by the presence of $\mathrm{H}$, and for sufficiently high $\mathrm{H}$ pressure a new $2 \times 2$ surface structure that is believed to be H-terminated is observed. Under Ga-rich conditions, the presence of hydrogen is found to induce step bunching on the surface, from which we argue that $\mathrm{H}$ selectively bonds to surface step and/or kink sites.

(C) 2003 WILEY-VCH Verlag GmbH \& Co. KGaA, Weinheim

1 Introduction The basic growth techniques for GaN films can be divided between those in which abundant hydrogen is present (e.g. metal organic chemical vapor deposition (MOCVD) [1] and reactive molecular beam epitaxy (RMBE) using ammonia [2]) and those without intentionally introduced hydrogen (e.g. plasma-assisted MBE, PAMBE [3]). The surface structure of GaN films grown by PAMBE are quite well understood due to the relative ease in carrying out in-situ surface studies, with the main surface structure of Ga-polar films grown under Ga-rich conditions consisting of about 2 monolayers (1 bilayer) of Ga on the surface (i.e. the "1×1" surface) [3]. Surface studies for MOCVD films also exist [4]. Studies $[5,6]$ have been carried out focusing on the dependence of growth rate of GaN films (of both polarities) in the presence of $\mathrm{H}$. Theoretical work has also been performed to investigate the effect of $\mathrm{H}$ on $\mathrm{GaN}$ growth $[7,8]$. In this work, we study the $\mathrm{GaN}(0001)$ surface structure in the presence of $\mathrm{H}$, in an effort to improve understanding of the link between MOCVD/RMBE and PAMBE growth.

In the absence of $\mathrm{H}$, there is a well known smooth-to-rough transition during PAMBE when the Ga-flux is reduced, during which the surface structure changes from the Ga-rich " $1 \times 1$ " to a Ga-poor surface terminated by Ga- or N-adatoms [3]. We find in the present work that, for relatively low pressure of $\mathrm{H}$, this transition persists but the critical Ga flux at which it occurs increases as the $\mathrm{H}$ pressure increases. We argue, following the work of $\mathrm{Yu}$ et al. and Van Mil et al. [5,6], that the $\mathrm{H}$ is modifying the gas phase or surface adsorption kinetics and producing more active $\mathrm{N}$ for the $\mathrm{GaN}$ growth. For larger $\mathrm{H}$ pressures and under Ga-poor conditions we observe a new surface structure, appearing as a sharp $2 \times 2$ pattern in reflection high-energy electron diffraction (RHEED). We tentatively associate this surface with the $\mathrm{N}_{\mathrm{ad}}-\mathrm{H}+\mathrm{Ga}-\mathrm{H}$ structure predicted by Van de Walle et al. [7]. Under Ga-rich conditions with the " $1 \times 1$ " present, the effect of hydrogen is to modify the surface morphology: Step bunching is observed, with the formation of 6-12 $\mathrm{nm}$ high steps on the surface. Subsequent annealing of this step-bunched surface produces small surface depressions, similar to those commonly reported for the MOCVD GaN and associated with dislocations intersecting the surface [1,9].

2 Experimental Our $\mathrm{GaN}$ growth is performed with a RF-plasma source of $\mathrm{N}$ with a fixed $\mathrm{N}_{2}$ partial pressure of $2 \times 10^{-5}$ Torr and using an effusion cell for $\mathrm{Ga}$, in an ultra-high vacuum (UHV) growth

\footnotetext{
${ }^{*}$ Corresponding author: e-mail: feenstra@cmu.edu, Phone: +1 412268 6961, Fax: +1 4126810648
} 
chamber equipped with RHEED. Hydrogen gas is introduced through the RF-plasma source. The spectral line of atomic hydrogen is observed from optical spectra of the plasma suggesting that some of the $\mathrm{H}_{2}$ molecules have dissociated, but the exact percentage of that is unknown. All growths discussed here were performed at $780^{\circ} \mathrm{C}$. Substrates consist of either H-etched $6 \mathrm{H}-\mathrm{SiC}(0001)$ (Si-polar) wafers or commercially available $\mathrm{GaN}(0001)$ (Ga-polar) films on sapphire (grown by hydride vapor phase epitaxy, HVPE), both without intentional miscut. Auger electron spectroscopy (AES) and low energy electron diffraction (LEED) is carried out in a UHV analysis chamber directly adjoined with growth chamber. The AES is performed with an incident electron beam energy of $3 \mathrm{keV}$, and using the Ga $(1070 \mathrm{eV})$ and $\mathrm{N}(379 \mathrm{eV})$ peaks to calculate a $\mathrm{Ga} / \mathrm{N}$ ratio. Surface morphology study is carried out using ex-situ atomic force microscope (AFM).

3 Results and Discussion The circular data points in Fig. 1(a) display the critical Ga flux at which a streaky-to-spotty transition in RHEED (i.e. smooth-to-rough transition in morphology) occurs, for various values of the $\mathrm{H}$ pressure. We also plot corresponding values of the $\mathrm{H}$ chemical potential, $\mu_{\mathrm{H}}$, computed according to the method of Ref. [7], in which $\mu_{\mathrm{H}}$ is determined using results for an ideal gas of $\mathrm{H}_{2}$ molecules at specified temperature and pressure. This method does not consider the effect of plasma and non-equilibrium nature of $\mathrm{MBE}$ on $\mu_{\mathrm{H}}$, so there may be some uncertainty in comparing our $\mu_{\mathrm{H}}$ values with the theoretical ones [7].
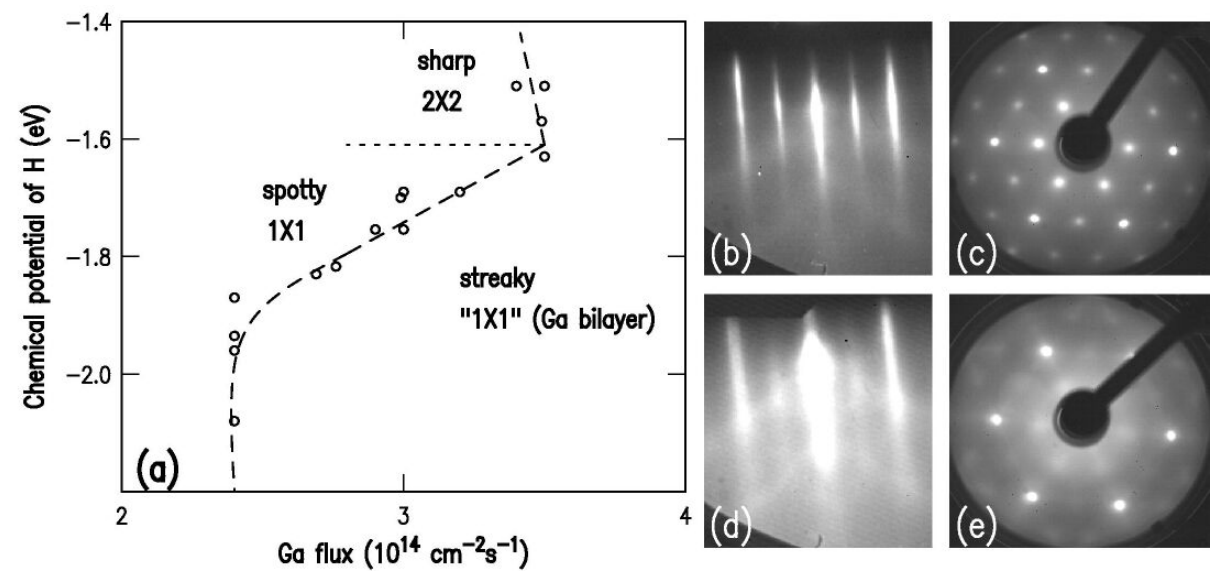

Fig. 1 (a) Dependence of the streaky-to-spotty Ga flux transition point (circles) on the chemical potential of hydrogen. The dashed line is drawn as a guide to the eye. The dotted line is an illustrative boundary between the two phases (Exact measurement of

this line is not possible since the sharp $2 \times 2$ becomes spotty after some time). (b), (c) RHEED pattern along [11 $\overline{2} 0]$ azimuth and LEED pattern ( $82 \mathrm{eV}$ electron energy) of sharp $2 \times 2$ obtained with the presence of hydrogen. (d), (e) RHEED pattern along [11 20$]$ azimuth and LEED pattern ( $84 \mathrm{eV}$ electron energy) of diffuse $2 \times 2$ obtained by exposure to pure $\mathrm{N}_{2}$.

On the Ga-rich side of Fig. 1(a), RHEED, LEED and Auger spectroscopy all exhibit a " $1 \times 1$ " Gabilayer surface termination characteristics. For $\mu_{\mathrm{H}}$ values below about $-1.6 \mathrm{eV}$, the streaky-to-spotty transition point shifts to higher Ga fluxes as $\mu_{\mathrm{H}}$ increases. On the Ga-poor side of the transition we observe the same type of spotty $1 \times 1$ RHEED pattern as seen in the absence of $H$. However when $\mu_{H}$ reaches about $-1.6 \mathrm{eV}$ this behavior stops, and instead we observe on the Ga-poor side of the transition a new surface structure, with a clear sharp, streaky $2 \times 2$ RHEED pattern developing during the growth. This "sharp $2 \times 2$ " is much sharper than the diffuse $2 \times 2$ RHEED pattern which is obtained by exposing surface to pure $\mathrm{N}_{2}$ plasma [10]. As the growth continues this sharp $2 \times 2$ pattern slowly begins to become spotty, indicating roughing of the surface under this condition. A stable streaky $2 \times 2$ could not be obtained, suggesting that this surface may be different from the $2 \times 2$ surface observed by ammonia-assisted MBE [2]. We note that this sharp $2 \times 2$ pattern can also be obtained by a second preparation method using growth interruption: We close the Ga shutter when growth is in streaky " $1 \times 1$ " RHEED region and ex-

(C) 2003 WILEY-VCH Verlag GmbH \& Co. KGaA, Weinheim 
pose the surface to the mixed $\mathrm{H}_{2}+\mathrm{N}_{2}$ plasma. The "sharp $2 \times 2$ " then appears. This process requires a much lower $\mu_{\mathrm{H}}$ compared with the first method. AES on these two kinds of surface prepared by different methods gives out same $\mathrm{Ga} / \mathrm{N}$ ratio within error bar $(\mathrm{Ga} / \mathrm{N}=0.86 \pm 0.03$ in both cases).

To summarize, there are two findings reflected from Fig. 1(a). First, the streaky-to-spotty transition point shifts towards higher Ga flux when the abundance of hydrogen increases (but not too much hydrogen). This result is consistent with observation of VanMil et al., who suggest that that atomic nitrogen atoms are a relatively inefficient species for the growth of $\mathrm{GaN}$ [6]. In the presence of $\mathrm{H}$ however, these species can be efficiently captured perhaps by forming surface adsorbed species similar to those occurring during $\mathrm{RMBE}$ growth with ammonia $\left(\mathrm{NH}_{3}\right)$ [2]. Thus, the presence of hydrogen is expected to increase the amount of active $\mathrm{N}$ species available for GaN growth. In this scenario, $\mathrm{H}$ does not form bonds directly with GaN surface, but instead it affects the gas species near the growth interface and/or the surface adsorbed species. In contrast, when there is enough hydrogen ( $\mu_{\mathrm{H}}$ approaching $\left.-1.6 \mathrm{eV}\right)$, it begins to bond with surface directly, consistent with the theoretical predictions of Van de Walle et al. [7]. Since hydrogen now can bond with surface, the amount of hydrogen in the gas vapor is correspondingly reduced. The amount of ammonia formed is also reduced. Thus the increasing dependence of the Ga flux transition point with $\mathrm{H}$ pressure can no longer be maintained. This change of slope of the transition line is apparent in the Fig. 1(a).

The second result from Fig. 1(a) is that a "sharp 2×2" RHEED pattern shows up. Figs. 1(b) and (c) shows the RHEED and LEED pattern of that surface. Comparing to the Ref. [7], we tentatively associate this surface with the $\mathrm{N}_{\mathrm{ad}}-\mathrm{H}+\mathrm{Ga}-\mathrm{H}$ structure. Since hydrogen atoms do not have significant effects on Auger spectroscopy, it is expected that this $\mathrm{N}_{\mathrm{ad}}-\mathrm{H}+\mathrm{Ga}-\mathrm{H}$ surface will have same $\mathrm{Ga} / \mathrm{N}$ Auger ratio as a $\mathrm{N}$-adatom surface. Within experimental error, the measured $\mathrm{Ga} / \mathrm{N}$ Auger ratio is same for this "sharp $2 \times 2$ " surface and nitrided surface prepared by pure $\mathrm{N}_{2}$ plasma exposure [3] $(\mathrm{Ga} / \mathrm{N}=0.86 \pm 0.03)$.

Figure 2(a) and (b) show AFM images of a film grown in the presence of $\mathrm{H}\left(\mathrm{H}_{2}\right.$ partial pressure of $2 \times 10^{-6}$ Torr). Step bunching is clearly visible in these images and also in the line cut of Fig. 2(c), whereas a film grown without $\mathrm{H}$ (Fig. 2(d)) does not display bunching. Typical step heights in Fig. 2(b) are found to be $6-12 \mathrm{~nm}$, as compared to the unit cell height of GaN of $0.52 \mathrm{~nm}$, although larger step bunches are also observed (e.g. as surrounds the small hexagonal terrace seen in the upper part of Fig. 2(b), with step height of $90 \mathrm{~nm}$ ). Actually, the latter large-step-height bunches are more predominant on samples grown on $\mathrm{SiC}$, and the 6-12 $\mathrm{nm}$ high bunches may be influenced by screw-type dislocations with a Burgers vector of this same magnitude, present on the HVPE-grown substrates as in Fig. 2. It should also be noted that within each terrace separated by the step bunches, small unit-cell high steps still occur. Step bunching has been previously observed for clean, vicinal GaN (0001) and H-exposed GaAs surfaces [11-13]. In the present case of H-exposed $\mathrm{GaN}$, it appears likely $\mathrm{H}$ preferentially bonds to the facet walls of the step bunches, i.e. those H-terminated faces have a low surface energy. We also note that the step edge of bunched steps is much longer (larger radius of curvature) compared with that of unit-cell high steps. Relatively large spirals formed by those step bunches are visible in large-area image of Fig. 2(b).

Figure 2(e) shows an AFM image of a step-bunched sample that has undergone a subsequent threeminute $\mathrm{N}_{2}+\mathrm{H}_{2}$ plasma exposure at $780^{\circ} \mathrm{C}$. The new features are small surface depressions emerging in the flat terrace, as shown in Fig. 2(f). The lateral dimension of these depressions at the surface is around 0.1 $\mu \mathrm{m}$. The depth of depressions varies from 2 to $6 \mathrm{~nm}$. Studies of MOCVD-grown GaN films have found similar surface depressions and attribute them to the dislocations intersecting the surface [1,9]. For the sample here, it is likely that those surface depression valleys are filled with metallic Ga before annealing, as observed by Hsu et al. [14]. Upon annealing in the presence of $\mathrm{H}$, the Ga evaporates and steps surrounding the depression become H-terminated, generating a MOCVD-like behavior there. In short, from the appearance of step bunching and surface depressions, the locations at which hydrogen begin to bond are probably non-(0001) surface sites, such as step edges or facets around dislocation cores. On those sites, either there is less Ga coverage or the energetics prefers hydrogen termination over Ga.

4 Conclusions We have studied the effect of introducing hydrogen gas through the RF plasma source during PAMBE of Ga-polar GaN(0001). It is found a transition from the " $1 \times 1$ " Ga-bilayer surface to one 

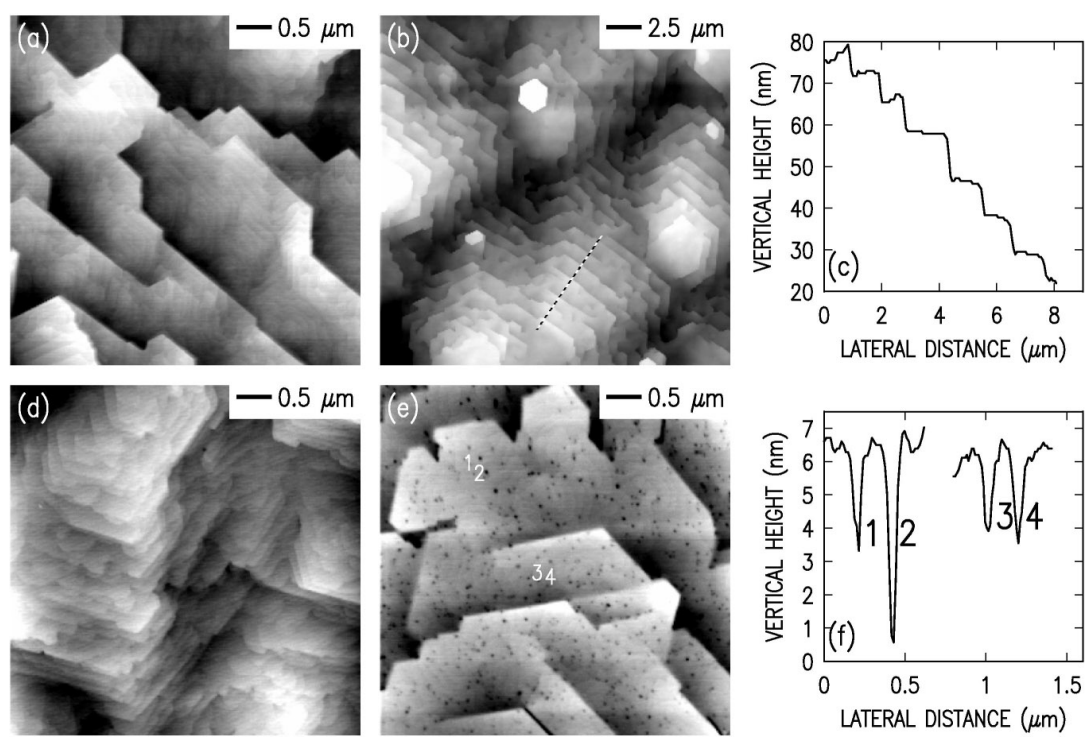

Fig. 2 (a) and (b) AFM images of $\mathrm{GaN}$ films grown in the presence of $\mathrm{H}$ (gray scales ranges 11 and $46 \mathrm{~nm}$, respectively). (c) Line cut along the dashed line in (b). (d) AFM image of GaN film grown with pure $\mathrm{N}_{2}$ (gray scale $8 \mathrm{~nm}$ ). (e) AFM image of $\mathrm{GaN}$ film grown in the presence of $\mathrm{H}$, that has undergone an additional 3 $\min \mathrm{N}_{2}+\mathrm{H}_{2}$ plasma exposure at $780^{\circ} \mathrm{C}$ (gray scale 10 $\mathrm{nm}$ ). (f) Line cuts of four surface depressions, with their identifying numbers (1-4) shown directly below each depression in (e). All films are grown on HVPEGaN-on-sapphire substrates.

displaying a sharp $2 \times 2$ pattern occurs when the chemical potential of $\mathrm{H}\left(\mu_{\mathrm{H}}\right)$ reaches about $-1.6 \mathrm{eV}$. We tentatively associated this $2 \times 2$ surface with the $\mathrm{N}_{\mathrm{ad}}-\mathrm{H}+\mathrm{Ga}-\mathrm{H}$ surface predicted by Van de Walle et al.. This surface is found to produce a rough growth morphology. For the " $1 \times 1$ " Ga-bilayer surface, $\mathrm{H}$ is found to induce step bunching and surface depressions, suggesting that the $\mathrm{H}$ bonds most actively to sites such as step edges and locations around dislocation cores.

Acknowledgements We gratefully acknowledge discussions with T. H. Myers and J. E. Northrup and assistance from S. Gaan on the H-etching of SiC samples. This work has been supported by the Office of Naval Research, Grant N00014-02-1-0933 monitored by C. Wood.

\section{References}

[1] B. Heying, E. J. Tarsa, C. R. Elsass, P. Fini, S. P. DenBaars, and J. S. Speck, J. Appl. Phys., 85, 6470 (1999).

[2] A. Thamm, O. Brandt, Y. Takemura, A. Trampert, and K. H. Ploog, Appl. Phys. Lett., 75, 944 (1999).

[3] A. R. Smith, R. M. Feenstra, D. W. Greve, M. -S. Shin, M. Skowronski, J. Neugebauer, and J. E. Northrup, J. Vac. Sci. Technol., B16, 2242 (1998).

[4] A. Munkholm, G. B. Stephenson, J. A. Eastman, C. Thompson, P. Fini, J. S. Speck, O. Auciello, P. H. Fuoss, and S. P. DenBaars, Phys. Rev. Lett., 83, 741 (1999).

[5] Zhonghai Yu, S. L. Buczkowski, N. C. Giles, T. H. Myers, and M. R. Richards-Babb, Appl. Phys. Lett., 69, 2731 (1996).

[6] B. L. VanMil, H. Guo, L. J. Holbert, K. Lee, T. H. Myers, T. Liu, and D. Korakakis, J. Cryst. Growth, (2004), (in press).

[7] Chris G. Van de Walle and J. Neugebauer, Phys. Rev. Lett., 88, 066103 (2002).

[8] John E. Northrup, Phys. Rev. B., 66, 045204 (2002).

[9] A. Krtschil, A. Dadgar, and A. Krost, J. Cryst. Growth, 248, 542 (2003).

[10] A. R. Smith, V. Ramachandran, R. M. Feenstra, D. W. Greve, A. Ptak, T. H. Myers, W. Sarney, L. SalamancaRiba, M. Shin, and M. Skowronski, MRS Internet J. Nitride Semicond. Res., 3, 12 (1998).

[11] M. H. Xie, S. H. Cheung, L. Zheng, Y. Ng, H. Wu, N. Ohtani, and S. Y. Tong, Phys. Rev. B., 61, 9983 (2000).

[12] M. V. Ramana Murty, P. Fini, G. B. Stephenson, C. Thompson, J. A. Eastman, A. Munkholm, O. Auciello, R. Jothilingam, S. P. DenBaars, and J. S. Speck, Phys. Rev. B., 62, 10661 (2000).

[13] R. Nötzel, H. Schönherr, Z. Nui, L. Däweritz, and K. H. Ploog, J. Crys. Growth., 201, 814 (1999).

[14] J. W. P. Hsu, M. J. Manfra, S. N. G. Chu, C. H. Chen, L. N. Pfeiffer, and R. J. Molnar, Appl. Phys. Lett., 78, 3980 (2001). 